\title{
The Use of Fuzzy Logic in the Enterprise's Fundamental Strength Assessment
}

\author{
Tomasz L. Nawrocki \\ Faculty of Organization and Management, Institute of Economics and Informatics \\ Silesian University of Technology \\ Zabrze, Poland \\ Tomasz.nawrocki@polsl.pl.
}

\begin{abstract}
The main aim of the paper is to present a practical application of an original enterprise's fundamental strength assessment fuzzy model, as an alternative to dominant in the literature solutions based on taxonomic methodology. Proposed model is an attempt to combine the fuzzy methodology with financial ratios (both in static and dynamic form) and in contrast to the most popular solutions in this area, it covers wider range of enterprise's performance dimensions, in particular financial condition, financial results, cash flows and operating efficiency, as well as development potential. Such a comprehensive approach to enterprise's fundamental strength assessment makes it more resistant to changes of business conditions, and thus should be more objective in comparison with taxonomic models mostly based on several, often limited in terms of information, financial ratios. In addition, it should be noted, that the use of fuzzy logic allows for significant flexibility in both - the modelcreating step and during its later use. As a result, the proposed solution is relatively easy to adapt to a particular specificity of enterprises and can be further modified/developed. Practical application of the model has been presented in the paper on the example of IT companies listed on the Warsaw Stock Exchange.
\end{abstract}

Keywords-Enterprise's fundamental strength; Financial ratios; Fuzzy logic

\section{INTRODUCTION}

Motivation to determine an overall company's fundamental strength assessment can be intuitively associated on the one hand with investment issues and, on the other hand, managerial issues. In the first case, it is about identifying the most interesting entities, that stand out positively in terms of broadly understood econo-financial situation. In the second, about assessment of business management quality.

The genesis of the enterprise's fundamental strength term is naturally connected with fundamental analysis [1][2], although some features are common also with the concept of value management [3]. Statement that company is fundamentally strong, in the understanding of an investor or analyst, is subconsciously identified with a well-run business, with good economic and financial standing and a favourable perception on the capital market, especially in longer term [4]. However, the problem is to translate this understanding into a model. In academic considerations can indeed be spotted some attempts in this regard (in both cases taxonomic methodology is used), but it is difficult recognize these solutions as complete and objective ones, due to either the lack of clear assessment criteria and empirical verification [5], or the use of fairly vague and superficial criteria [6].

Therefore, the main aim of the paper is to present an exemplary application of developed fuzzy model, which general concept was already published by the author [7].

\section{RESEARCH METHODOLOGY}

In the proposed solution [7], a company with a high fundamental strength should distinguish itself with permanent ability to generate good quality financial results and cash flows, high efficiency and operating performance, good financial condition and high level of development potential, both material and intangible, including human capital.

Assessment criteria in this solution were selected in three thematic areas - financial condition, business results and efficiency, development potential (Table I) - by analysing specific characteristics of many financial ratios used in the corporate financial analysis [2][8], taking into account rather their quality/information than popularity as well as author's knowledge and years of practical experience in the field of fundamental analysis in Stockwatch.pl.

TABLE I. Fundamental StRENGTH ASSESSMENT CRITERIA

\begin{tabular}{|c|c|}
\hline $\begin{array}{c}\text { Thematic } \\
\text { area }\end{array}$ & Assessment criteria \\
\hline \multirow{4}{*}{$\begin{array}{l}\text { Financial } \\
\text { condition }\end{array}$} & Quick Ratio \\
\hline & Short-term Debt Coverage by Operating Cash Flow \\
\hline & Financial Debt - Equity Ratio \\
\hline & Net Financial Debt - Net Profit from Sales and Amort. Ratio \\
\hline \multirow{8}{*}{$\begin{array}{l}\text { Business } \\
\text { results and } \\
\text { efficiency }\end{array}$} & Net Profit y/y Growth \\
\hline & Quality of Net Profit \\
\hline & Cash (End of Cash Flows) y/y Growth \\
\hline & Impact of Net Financial Surplus on Total Cash Flows \\
\hline & Net Margin on Sales \\
\hline & Return on Equity \\
\hline & Difference between Net Work. Capital and Cash Conv. Cycle \\
\hline & Cash Conversion Cycle \\
\hline \multirow{6}{*}{$\begin{array}{l}\text { Development } \\
\text { potential }\end{array}$} & Employee Valuation \\
\hline & Work Efficiency \\
\hline & Fixed and Intangible Assets Depreciation Ratio \\
\hline & Fixed and Intangible Assets y/y Growth \\
\hline & Results of R\&D Activity \\
\hline & Intensity of Investment Expenditures \\
\hline
\end{tabular}


This criteria within the structure of the model, which was published earlier [7], are supposed to be aggregated to more and more general modules with the final module assessing the enterprise's fundamental strength ending.

The calculation tool in the suggested solution is based on the fuzzy logic invented by L.A. Zadeh [9], which uses an approximate reasoning.

It was also assumed, that financial data for calculation purposes will be obtained from financial reports of analyzed companies. In all cases of variables based on balance sheet (financial situation statement) values it is also assumed to use their average values from two-year period.

Additionally, to take into account within the individual input variables of the model their current level and lately tendency ( 2 years back), their calculation is supposed to be performed on the basis of weighted average formula [7]. This type of an approach makes, that the final enterprise's fundamental strength assessment is less susceptible to fluctuations caused by changes in the value of basic evaluation criteria. It is also, in a sense, a way to appreciate entities, which develop less chaotic and more stable. It was decided to include two periods backwards because in such situation there is a balance of influence on the input variable final evaluation by current value of given partial criterion and its readings from earlier periods. At one retrograde time $(n=2)$, it would be a prevalence of current reading, and at three or more back periods $(n \geq 4)$ - earlier readings.

In relation to the construction process of proposed fuzzy model [7] the Mamdani approach [10] was used and some assumptions were made regarding its individual stages:

- for all input variables of the model $\left(x_{i}\right)$, the same dictionary of linguistic values was used, and their value space was divided into three fuzzy sets named \{low, medium, high\};

- for output variables of the model $(y)$, in order to obtain more accurate intermediate assessments, the space of linguistic values was divided into five fuzzy sets named \{low, mid-low, medium, mid-high, high\};

- in case of all membership functions to the particular fuzzy sets, a triangular shape was decided for them (Fig. 1. and Fig. 2.);

- the values of fuzzy sets characteristic points $(x 1, x 2, x 3)$ for the particular input variables of the model were determined partly basing on the literature on corporate economic and financial analysis and partly arbitrarily basing on author years of experience in the area of fundamental analysis and distribution of values for the investigated companies (Table II);

- for fuzzification of input variables, the method of simple linear interpolation was used [11];

- fuzzy reasoning in the particular knowledge bases of the model was conducted using PROD operator (fuzzy implication) and SUM operator (final accumulation of the conclusion functions received within the particular rule bases into one output set for each base) [11];
- for defuzzification of fuzzy reasoning results Simplified Centre of Sums Method was used [11].

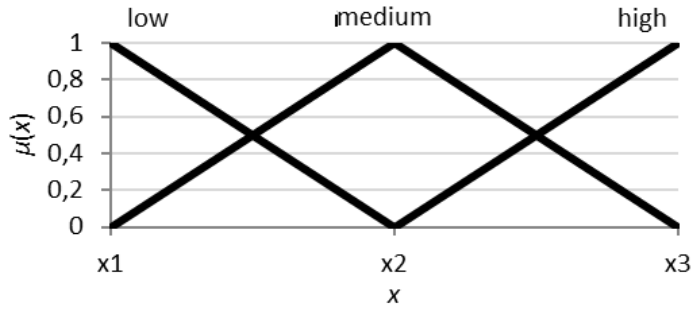

Fig. 1.The input variables membership function.

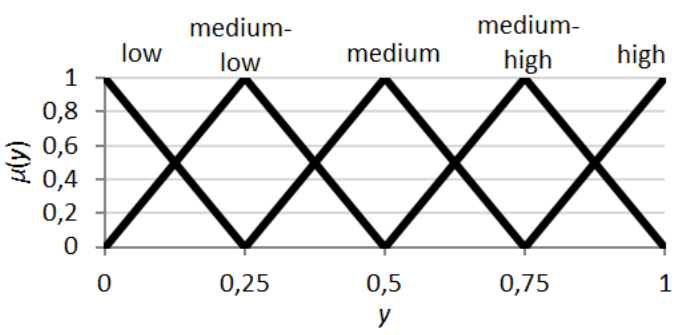

Fig. 2. The output variables membership function.

Next, taking into consideration general structure of the model [7], author, basing on his knowledge and experience in the area of analyzed issue, designed 16 rules bases in the form of ,IF - THEN" sentences (15 bases with 9 rules and 1 base with 27 rules), achieving this way ,ready to use” form of the original (authorial) fundamental strength assessment fuzzy model. Due to a large volume of all knowledge (rules) bases included in the proposed model, below has been presented only the last one of them.

Rules base for assessment in the area of the final enterprise's fundamental strength - AFS (AFC - financial condition assessment, ABRE - business results and efficiency assessment, ADP - development potential assessment; $\mathrm{L}$ - low, $\mathrm{M}$ - medium, $\mathrm{H}$ - high, $\mathrm{ML}$ - mid-low, $\mathrm{MH}$ - mid-high):

R1: IF $A F C$ is L AND ABRE is L AND ADP is $\mathrm{L}$ THEN AFS is $\mathrm{L}$ R2: IF $A F C$ is L AND $A B R E$ is L AND $A D P$ is M THEN AFS is $\mathrm{L}$ R3: IF $A F C$ is $L$ AND $A B R E$ is M AND ADP is L THEN AFS is $\mathrm{L}$ R4: IF $A F C$ is M AND ABRE is L AND ADP is L THEN AFS is L R5: IF $A F C$ is L AND $A B R E$ is M AND ADP is M THEN AFS is ML R6: IF $A F C$ is M AND ABRE is M AND ADP is L THEN AFS is ML R7: IF AFC is M AND ABRE is L AND ADP is M THEN AFS is ML R8: IF $A F C$ is L AND ABRE is L AND ADP is H THEN AFS is ML R9: IF $A F C$ is L AND ABRE is H AND $A D P$ is L THEN AFS is ML R10: IF $A F C$ is H AND $A B R E$ is L AND $A D P$ is L THEN AFS is ML R11: IF $A F C$ is M AND ABRE is L AND ADP is H THEN AFS is ML R12: IF $A F C$ is H AND $A B R E$ is L AND $A D P$ is M THEN AFS is ML R13: IF $A F C$ is M AND ABRE is M AND ADP is M THEN AFS is M R14: IF $A F C$ is L AND ABRE is H AND ADP is $\mathrm{H}$ THEN AFS is $\mathrm{M}$ R15: IF AFC is H AND ABRE is H AND ADP is L THEN AFS is M R16: IF $A F C$ is H AND ABRE is L AND ADP is H THEN AFS is M R17: IF $A F C$ is $H$ AND $A B R E$ is $M$ AND $A D P$ is M THEN AFS is $M$ R18: IF $A F C$ is L AND $A B R E$ is M AND $A D P$ is H THEN AFS is M R19: IF $A F C$ is L AND $A B R E$ is H AND $A D P$ is M THEN AFS is M R20: IF $A F C$ is M AND ABRE is H AND ADP is L THEN AFS is M R21: IF AFC is H AND ABRE is M AND ADP is L THEN AFS is M R22: IF $A F C$ is M AND ABRE is M AND ADP is H THEN AFS is MH R23: IF $A F C$ is M AND ABRE is H AND ADP is M THEN AFS is MH R24: IF $A F C$ is H AND ABRE is M AND $A D P$ is $\mathrm{H}$ THEN AFS is $\mathrm{MH}$ R25: IF AFC is M AND ABRE is H AND ADP is H THEN AFS is $\mathrm{H}$ R26: IF $A F C$ is H AND $A B R E$ is $\mathrm{H}$ AND $A D P$ is M THEN AFS is $\mathrm{H}$ R27: IF $A F C$ is $\mathrm{H}$ AND $A B R E$ is $\mathrm{H}$ AND $A D P$ is $\mathrm{H}$ THEN $A F S$ is $\mathrm{H}$ 
TABLE II. THE VALUES OF FUZZY SETS CHARACTERISTIC POINTS FOR PARTICULAR INPUT VARIABLES OF ENTERPRISE'S FUNDAMENTAL STRENGTH ASSESSMENT FUZZY MODEL

\begin{tabular}{|c|c|c|c|c|}
\hline Symbol & Name & $\begin{array}{c}x 1 \\
\mu(x)=1 / \text { low }\end{array}$ & $\begin{array}{c}x 2 \\
\mu(x)=1 / \text { medium }\end{array}$ & $\begin{array}{c}x 3 \\
\mu(x)=1 / \text { high } \\
\end{array}$ \\
\hline A & Quick Ratio [-] & 0 & 0,75 & 1,5 \\
\hline $\mathrm{B}$ & Short-term Debt Coverage by Operating Cash Flow [-] & 0 & 0,75 & 1,5 \\
\hline $\mathrm{C}$ & Financial Debt - Equity Ratio [-] & 0 & 1 & 2 \\
\hline $\mathrm{D}$ & Net Financial Debt - Net Profit from Sales and Amortization Ratio [years] & 0 & 3 & 6 \\
\hline E & Net Profit y/y Growth (\%) & -50 & 0 & 50 \\
\hline $\mathrm{F}$ & Quality of Net Profit [-] & 0 & 0,5 & 1 \\
\hline G & Cash (End of Cash Flows) y/y Growth [\%] & -50 & 0 & 50 \\
\hline $\mathrm{H}$ & Impact of Net Financial Surplus on Total Cash Flows [-] & 0 & 0,6 & 1,2 \\
\hline $\mathrm{I}$ & Net Margin on Sales [\%] & 0 & 15 & 30 \\
\hline $\mathrm{J}$ & Return on Equity [\%] & 0 & 10 & 20 \\
\hline $\mathrm{K}$ & Difference between Net Working Capital and Cash Conversion Cycle [days] & -30 & 15 & 60 \\
\hline $\mathrm{L}$ & Cash Conversion Cycle [days] & -30 & 15 & 60 \\
\hline $\mathrm{M}$ & Employee Valuation [000\%/employee] & 0 & 50 & 100 \\
\hline $\mathrm{N}$ & Work Efficiency [000\%/employee] & 0 & 125 & 250 \\
\hline $\mathrm{O}$ & Fixed and Intangible Assets Depreciation Ratio [-] & 0,4 & 0,7 & 1 \\
\hline $\mathrm{P}$ & Fixed and Intangible Assets y/y Growth [\%] & -20 & 0 & 20 \\
\hline $\mathrm{R}$ & Results of R\&D Activity [-] & 0 & 0,5 & 1 \\
\hline $\mathrm{S}$ & Intensity of Investment Expenditures [\%] & 0 & 5 & 10 \\
\hline
\end{tabular}

The intermediate and final assessments generated by the model take values in the range between 0 and 1 , where from the viewpoint of analyzed issue, values closer to 1 mean a more favorable result, while values closer to 0 a less favorable.

All calculations related to the presented fuzzy model were based on self-developed structure of formulas in MS Excel.

\section{RESEARCH RESULTS}

Exemplary application of developed enterprise's fundamental strength assessment fuzzy model was performed for five companies from IT industry involved in management support software creation, which shares were listed on the
Warsaw Stock Exchange in last three years - Asseco Business Solutions (Asseco BS), Comarch, LSI Software (LSI), Simple, Quantum Software (Quantum) - selection of these entities resulted from the comparability of their businesses. According to the adopted methodology, the basis for fundamental strength assessment of mentioned above entities were data acquired from their annual reports published in the years 2016-2018 (Table III).

Next, basing on weighted average formula [7], corrected values of particular input variables to the model for investigated companies were calculated. Final and intermediate results generated by the model were shown in Fig. 3 .

TABLE III. THE VALUES OF BASIC ASSESSMENT CRITERIA TO THE ENTERPRISE'S FUNDAMENTAL STRENGTH ASSESSMENT MODEL FOR INVESTIGATED COMPANIES

\begin{tabular}{|c|c|c|c|c|c|c|c|c|c|c|c|c|c|c|c|}
\hline & \multicolumn{3}{|c|}{ Asseco BS } & \multicolumn{3}{|c|}{ Comarch } & \multicolumn{3}{|c|}{ LSI } & \multicolumn{3}{|c|}{ Simple } & \multicolumn{3}{|c|}{ Quantum } \\
\hline & 2017 & 2016 & 2015 & 2017 & 2016 & 2015 & 2017 & 2016 & 2015 & 2017 & 2016 & 2015 & 2017 & 2016 & 2015 \\
\hline A & 0,85 & 4,05 & 4,06 & 1,69 & 1,79 & 1,74 & 1,38 & 1,50 & 1,54 & 0,91 & 0,87 & 0,80 & 3,08 & 4,37 & 4,72 \\
\hline B & 1,44 & 2,00 & 2,01 & 0,16 & 0,25 & 0,32 & 0,80 & 0,39 & 1,04 & 0,30 & 0,31 & 0,14 & 0,88 & 0,86 & 0,78 \\
\hline $\mathrm{C}$ & 0,12 & 0,00 & 0,00 & 0,38 & 0,22 & 0,19 & 0,13 & 0,13 & 0,09 & 0,31 & 0,35 & 0,72 & 0,02 & 0,01 & 0,03 \\
\hline $\mathrm{D}$ & 0,32 & 0,00 & 0,00 & 1,15 & 0,00 & 0,00 & 0,04 & 0,00 & 0,00 & 0,15 & 1,21 & 10,00 & 0,00 & 0,00 & 0,00 \\
\hline$E$ & $18,1 \%$ & $26,7 \%$ & $17,3 \%$ & $-42,5 \%$ & $-5,7 \%$ & $16,5 \%$ & $11,7 \%$ & $21,8 \%$ & $385,1 \%$ & $393,6 \%$ & $111,6 \%$ & $-175,4 \%$ & $11,8 \%$ & $23,3 \%$ & $262,1 \%$ \\
\hline $\mathrm{F}$ & 0,95 & 0,98 & 0,97 & 0,07 & 1,02 & 1,03 & 0,71 & 0,61 & 1,07 & 0,83 & 0,39 & $-0,96$ & 0,86 & 0,86 & 1,08 \\
\hline G & $-88,6 \%$ & $73,4 \%$ & $-29,3 \%$ & $-11,8 \%$ & $6,3 \%$ & $-4,6 \%$ & $-31,0 \%$ & $-6,0 \%$ & $486,2 \%$ & $452,3 \%$ & $24,3 \%$ & $-72,1 \%$ & $-17,2 \%$ & $12,8 \%$ & $32,9 \%$ \\
\hline $\mathrm{H}$ & 0,51 & 1,03 & 0,68 & 0,54 & 0,75 & 0,93 & 0,84 & 0,94 & 0,95 & 1,06 & 0,38 & $-0,33$ & 0,51 & 1,39 & 2,44 \\
\hline $\mathrm{I}$ & $41,2 \%$ & $43,9 \%$ & $36,0 \%$ & $3,5 \%$ & $11,5 \%$ & $11,6 \%$ & $11,3 \%$ & $12,8 \%$ & $21,5 \%$ & $8,5 \%$ & $2,3 \%$ & $-11,0 \%$ & $7,9 \%$ & $8,3 \%$ & $9,1 \%$ \\
\hline $\mathrm{J}$ & $17,2 \%$ & $15,4 \%$ & $12,4 \%$ & $0,4 \%$ & $9,4 \%$ & $11,2 \%$ & $11,9 \%$ & $10,6 \%$ & $17,9 \%$ & $16,7 \%$ & $1,9 \%$ & $-33,4 \%$ & $13,1 \%$ & $12,3 \%$ & $13,0 \%$ \\
\hline $\mathrm{K}$ & 52 & 147 & 148 & 46 & 64 & 64 & 15 & 38 & 22 & 1 & -29 & -19 & 101 & 94 & 81 \\
\hline $\mathrm{L}$ & 10 & 17 & 20 & 80 & 52 & 39 & 39 & 37 & 41 & -4 & 6 & 41 & 39 & 61 & 65 \\
\hline $\mathrm{M}$ & 121,89 & 118,61 & 109,08 & 123,64 & 127,37 & 119,61 & 74,93 & 68,54 & 66,47 & 74,87 & 66,35 & 69,97 & 79,94 & 70,60 & 65,19 \\
\hline $\mathrm{N}$ & 271,42 & 279,40 & 257,35 & 198,97 & 216,50 & 231,12 & 267,24 & 234,64 & 226,33 & 202,97 & 158,50 & 190,20 & 197,18 & 164,17 & 158,29 \\
\hline $\mathrm{O}$ & 0,56 & 0,71 & 0,73 & 0,48 & 0,48 & 0,48 & 0,57 & 0,56 & 0,57 & 0,68 & 0,68 & 0,61 & 0,76 & 0,70 & 0,61 \\
\hline $\mathrm{P}$ & $124,9 \%$ & $6,0 \%$ & $0,1 \%$ & $10,3 \%$ & $12,9 \%$ & $8,1 \%$ & $20,2 \%$ & $20,3 \%$ & $6,8 \%$ & $1,7 \%$ & $14,3 \%$ & $18,4 \%$ & $-6,7 \%$ & $-26,6 \%$ & $-27,7 \%$ \\
\hline $\mathrm{R}$ & 0,33 & 0,33 & 0,33 & 0,75 & 1,00 & 1,00 & 1,00 & 1,00 & 1,00 & 0,33 & 0,33 & 0,33 & 0,33 & 0,33 & 0,33 \\
\hline $\mathrm{S}$ & $6 \%$ & $7 \%$ & $7 \%$ & $13 \%$ & $11 \%$ & $8 \%$ & $10 \%$ & $11 \%$ & $17 \%$ & $6 \%$ & $10 \%$ & $8 \%$ & $2 \%$ & $1 \%$ & $1 \%$ \\
\hline
\end{tabular}




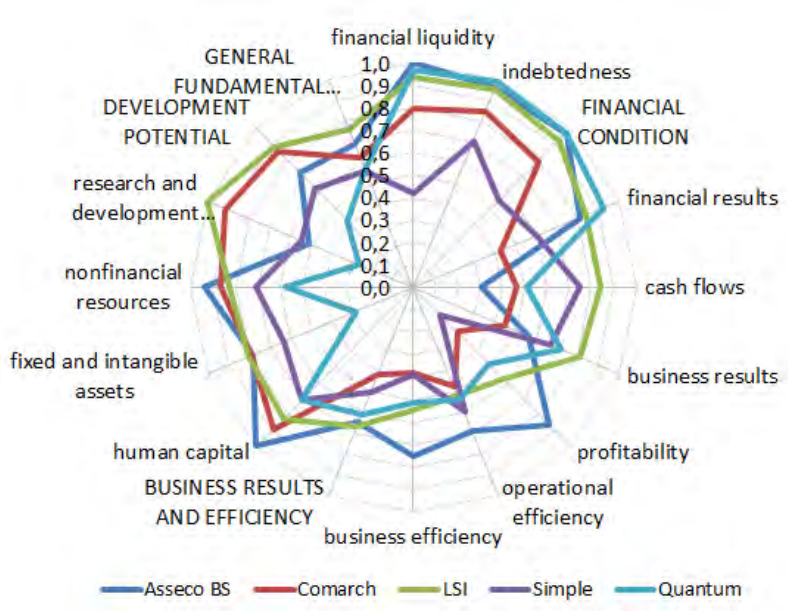

Fig. 3. Final and intermediate results of enterprise's fundamental strength assessment generated by the developed fuzzy model for investigated IT companies; results dated on 2017

Taking into account the results obtained, it can be stated that LSI Software was characterized by the greatest fundamental strength among the surveyed companies at the end of 2017 (0.759). Next places were taken by Asseco Business Solutions (0.692) and Comarch (0.625). In turn, the lowest marks, although still at a slightly above average level, were obtained for Quantum Software (0.572) and Simple (0.564). LSI was distinguished positively in all three thematic areas of general fundamental strength assessment financial condition (0.923), business results and efficiency $(0.670)$ as well as development potential (0.884), while Asseco Business Solutions stood out in terms of development potential (0.721), and Comarch in terms of financial condition (0.792) as well as business results and efficiency (0.416). When it comes to companies with lower ratings, Quantum Software, except for financial condition (0.972), was rather average (business results and efficiency 0.612 and development potential -0.420 ), while in turn Simple stood out negatively in particular in terms of financial condition (0.546).

\section{CONCLUSIONS}

The model of enterprise's fundamental strength assessment, developed using the methodology of fuzzy sets, is characterized by the following advantages, that partially enable reducing the defects of previously applied methods: (i) the use of full range financial indicators to provide greater objectivity of final results and less susceptible to falsify them on grounds of industry; (ii) combination of analytic and synthetic assessment, achieved through the use of subcriteria, that are next aggregated into a more general assessments; (iii) combination of quantitative and qualitative approach to the fundamental strength assessment, manifested mostly in a qualitative dimension of ratios used for its measurement and qualitative method of its diversification determination, but also in the use of qualitative criterion effects of R\&D activity; (iv) using for the assessment process data included in generally accessible companies' interim reports.
In the context of fuzzy logic use to analyze enterprises' fundamental strength it should be noted, that thereby assessments in individual thematic areas with different levels of aggregation were obtained, which on the one hand are consistent with the interpretation of analyzed companies situation on the basis of basic criteria/indicators, and on the other hand gave the possibility of better discernment in comparison with the processing of each criterion separately. It should be also noted that proposed model can be a useful tool supporting investment process in the stock market, as well as a tool supporting managerial decision being made in enterprises.

The concept of an enterprise's fundamental strength assessment fuzzy model presented in this paper has still a preliminary character. Its biggest controversy, one may say also weakness, is subjective selection of assessment criteria, mainly on the basis of Author's practical experience in the considered field. During further research presented model will be subject to certain modifications. They will cover in particular the issue of input variables value space division, to make it more universal and automatic (less arbitral).

\section{ACKNOWLEDGMENT}

This research was supported by BK-231/ROZ1/2018 (13/010/BK_18/0029).

\section{REFERENCES}

[1] B. Graham and D.L. Dodd, Securities Analysis. New York: McGrawHill Education Ltd. 2008.

[2] J. Ritchie, Fundamental Analysis: A Back to the basics. New York: McGraw-Hill, 1995.

[3] A. Rappaport, 10 Ways to Create Shareholder Value. Harvard Business Review, September 2006, pp. 66-77.

[4] J.D. Piotroski, "Value Investing: The Use of Historical Financial Statement Information to Separate Winners from Losers", Journal of Accounting Research, vol. 38, 2000, pp. 1-41.

[5] G. Mikołajewicz, The determinants of the fundamental strength of the company. Research Papers of the Wroclaw University of Economics, Issue 365, 2014, pp. 173-185.

[6] W. Tarczyński and M. Tarczyńska-Łuniewska, "Application of The Leading Sector Identification Method in The Portfolio Analysis," Acta Universitas Lodziensis. Folia Oeconomica, vol. 2(328), 2017, pp. 185-200.

[7] T.L. Nawrocki, "The concept of corporate fundamental strength assessment model based on fuzzy logic," In: P. Limpaphayom \& G. Huang, Proceedings of the Second International Conference On Economic and Business Management - FEBM-2017, AEBMR series, Atlantis Press, vol. 33, 2017, pp. 82-88.

[8] J. Lan, Financial Ratios for Analyzing a Company's Strengths and Weaknesses, 2012. Online: http://www.aaii.com/journal/article/16financial-ratios-for-analyzing-a-companys-strengths-and-weaknesses. touch (access: 20.04.2018).

[9] L.A. Zadeh, "Fuzzy sets," Information and Control. vol. 8(3), 1965, pp. 338-353.

[10] E.H. Mamdani and S. Assilian, "An experiment in linguistic synthesis with a fuzzy logic controller," International Journal of Man-Machine Studies, vol. 7(1), 1975, pp. 1-13.

[11] A. Piegat, Fuzzy Modeling and Control. Berlin Heidelberg: SpringerVerlag, 2001. 